\title{
Target Recognition of Software Research about Machine System of Accurately Spraying
}

\author{
Yan $\mathrm{Shi}^{1}$, Chunmei Zhang ${ }^{2}$, Maogang $\mathrm{Li}^{3}$, and Haibo Yuan ${ }^{4}$ \\ ${ }^{1}$ Department of Food Engineering, Qingdao Agriculture University, Qingdao, \\ Shandong Province, P.R. China 266109, Tel.: 0532-88030446 \\ shiyan517@163.com \\ ${ }^{2}$ Xian Air Force Engineering University, P.R. China \\ ${ }^{3}$ College of Mechanical \& Electrical Engineering, Qingdao Agricultural University, \\ Qingdao 266109, China \\ ${ }^{4}$ Ministry of Agricultural, The People's Republic of China, Beijing 100125, China
}

\begin{abstract}
The software is used modularity and qeneric idea, realize target recognition and accurately spraying, it consist of five modules: module of image process, module of identifing the crop from the background of elaphic, module of target recognition and classification of treatment, module of intelligent decision-making and helpness. Image process is made of distortion adjustment,gray strengthen, neighbourhoods average, histogram equalization and median filter; the module of identifing the crop from the background of elaphic is made of division of green strength threshold, division of $\mathrm{H}$ hue threshold and segmentation afterwards process and so on; the module of target recognition consist of recognition of fruiter, rice, wheate and target process and area calculating, the module of recognition of fruiter include centre recognition function; the module of intelligent decision-making include information target transmited. The system has fault-tolerant function and automatically judges the input of image or not, automatically detects input of camera and terminal setting, connection situation of singlechip, creating executable file, breaking away from $\mathrm{VC}++$ then direct running. Limiting surface of software system is friendly, simple clear and satisfy the real-time processing the request.
\end{abstract}

Keywords: precision employing pesticide, image process, the soft of recognition.

\section{Introduction}

Precision spraying technology are areas of precision agriculture, mainly refer to the help of computer vision technology, identify the weed coverage rate and determine the location of weeds. Accordingly the implementation of appropriate, positioning the spraying of herbicides, the absence of regional weed not spraying herbicides. This not only can effectively reduce weeds, improve yield and quality of agricultural products, 
but also significantly savings in the use of herbicides liang, lower input, protect the ecological environment, have important economic significance and ecological significance.

Many scholars have conducted extensive studies. Won Suk Lee from USA, Claim that they have developed a real-time and between the lines system used to control weeding intelligent robot[1-2]. M.E.R.Paice, P.C.H.Millerdesigned a injection metering system, by changing the mixing ratio of the method to adjust the liquid volume spraying[3-4]. May.Kenzel invented a spray bar height can be adjusted and automatically sprayed herbicides on the target machine system[5]. Nanjing Forestry University, Mao-Cheng Zhao design a system based on Tree features real-time accurate image of the pesticide on the target and can be variable spraying[6]. QIU Bai-jing from Jiangsu University developed a variable spray device, it can be installed in the hardware and software equipment and DGPS for guidance, according to their location automatically adjust the size of spray volume. The equipment includes sprayers, adjust the controller, the computer console, AgGPS132, farm tractors and other equipment[7].

Precision of the target application techniques one of the difficulties is the fast and accurate identification on the target, target image acquisition and processing are key part of machine vision, This article is used modularity and qeneric idea, realize target recognition and accurately spraying, it consist of five modules: module of image process, module of identifing the crop from the background of elaphic, module of target recognition and classification of treatment, module of intelligent decision-making and helpness.

\section{Image Acquisition and Preprocessing}

\subsection{Image Acquisition}

Realize dynamic acquisition with a CCD camera image in the actual system, image was real-time input computer through image acquisition card.

\subsection{Image Preprocessing}

As the spraying system image acquisition is in the outdoor, and the camera installed in the movement of locomotive, the collection of images often have many problems, such as the image edge is too vague and have some images made unexpected white spots or black spots, image distortion, affected by various natural conditions, such as wind, light, uneven exposure factors, the input image in the visual effects and identify possible have so many problems, these factors resulted in images "quality" problem. Therefore, the first pre-process the image. In the pre-processing stage, using distortion correction, gray transformation enhancement, histogram equalization algorithm, to improve the image quality. 


\section{Image Segmentation and Target Identification}

\subsection{Image Segmentation}

Making weed identification should be based on different backgrounds to choose a different recognition method. The weeds on the soil background recognition can be applied to spectrum and color of law, according to weed and background material in the color and spectral characteristics of the differences in segmentation. Green weed in the context of recognition are more complex, the background of the intensive plants, such as lawn, generally use shape feature method or texture feature method. Background of the drilling plant, to achieve the rapid division of weeds and crops with position characteristic; background-demand plants, the shape feature method is more used.

\subsection{Target Identification}

This study, on crop and background segmentation, used ultra-green method and tone method, in which the threshold was automatically determined, greater than the threshold value for the pixels to given the background color, less than the threshold value was given the same color of the pixel.

Such as drilling wheat crop planted artificially, so its position distribution is the rule - by line of distribution; and the growth of weeds is natural, non-regularly distributed between crop rows. In this study, the division of wheat and weeds used location characteristics method. Corn crop demand, $3 \sim 5$ leaves, has certain spacing and line spacing between adjacent plants, plant leaves overlap to a lesser. Therefore, for corn, the first extract their shape characteristics, and to train artificial neural network for recognition.

\subsection{Segmentation to Remove Noise}

The image segmented more or less has noise. The noise of the area generally consists of several pixels, the form of a point-like. By median filtering method to filter the image processing.

\subsection{Determine Target Area}

The binary image was divided into 24 squares and was statisticed (where the size of each small square region is just a liquid spray nozzle effective area), statistics for each small box the white pixels and the ratio of black pixels, the ratio is as the volume of the signal box region spray.

Binary image defined area A pesticide is sprayed area that contains the number of pixels. Hypothesis, the size of each square $\mathrm{b}(\mathrm{x}, \mathrm{y})$ equal $M \times N, b(x, y)=1$, the background is zero, the target area A:

$$
A=\sum_{x=0}^{M=1} \sum_{y=0}^{N=1} b(x, y)
$$




\section{Software System Design}

The system was used Microsoft Visual C++6.0 MFC to generate the basic framework, and then programmed with the Win32 API programming language, Microsoft has introduced the development of Win32 environment that is a visual integration of object-oriented programming environment. It is not only a procedural framework for automatic generation of flexible class management, coding and integration interaction interface design, can develop a variety of procedures (application, DLL, ActiveX controls, etc.), but also by a simple set can process framework to generate support for database interface, OLE2, Winsock network, 3D control interface.

\subsection{System Overview}

In the software process taking a good design is important. Rational design of software is not only development cycle short, low cost, but also readable, easy to maintain. On the contrary, free software written in is not only difficult to grasp, but also poor to read, hard to maintain, there may even be unable to complete the code and lead to the situation half-way scrap. The modular software design with high cohesion, low coupling principle of the software is divided into relatively independent of the number of parts in order to separate design and code of preparation, make the structure of the whole system easily understand. Have been introduced earlier in this article all aspects of the $\mathrm{VC}++$ programming, the use of modular programming, can take advantage of all the previous subroutine, after simple treatment can be integrated into the total program.

The software is divided into modularity through function, it consist of five modules: it consist of five modules: module of image process, module of identifing the crop from the background of elaphic, module of target recognition and classification of treatment, module of intelligent decision-making and helpness. Image process is made of distortion adjustment, gray strengthen, neighbourhoods average, histogram equalization and median filter; the module of identifing the crop from the background of elaphic is made of division of green strength threshold, division of $\mathrm{H}$ hue threshold and segmentation afterwards process and so on; the module of target recognition consist of recognition of fruiter, rice ,wheate and target process and area calculating, the module of recognition of fruiter include centre recognition function; the module of intelligent decision-making include information target transmited.

Program was used class-based ideology. In the program the images are a variety of operations and operations in the form of packaging up to class so that they can work for future expansion.

\subsection{System Framework}

The process is as follows:

(1) Frist, CCD images were collected, then were pretreatment, and binary image was processed with Green features. Crops and the background binary image was obtained. 
(2) The image was processed with morphological operations, eliminatie a lot of noise.

(3) Judgments are fruit trees, wheat or corn images into the appropriate processing module.

(a) There is tree, tree crown recognition.

(b) There is wheat, extracting centerline.

(c) There is crop, region marking, according to the distance between the region for regional consolidation, the feature of region of the combined was extracted, on the identification of maize, the input feature vectors extracted BP neural network classifier to judge whether the target.

(4) Calculate Target area and position.

(5) Send information.

Total system flow chart as follow:

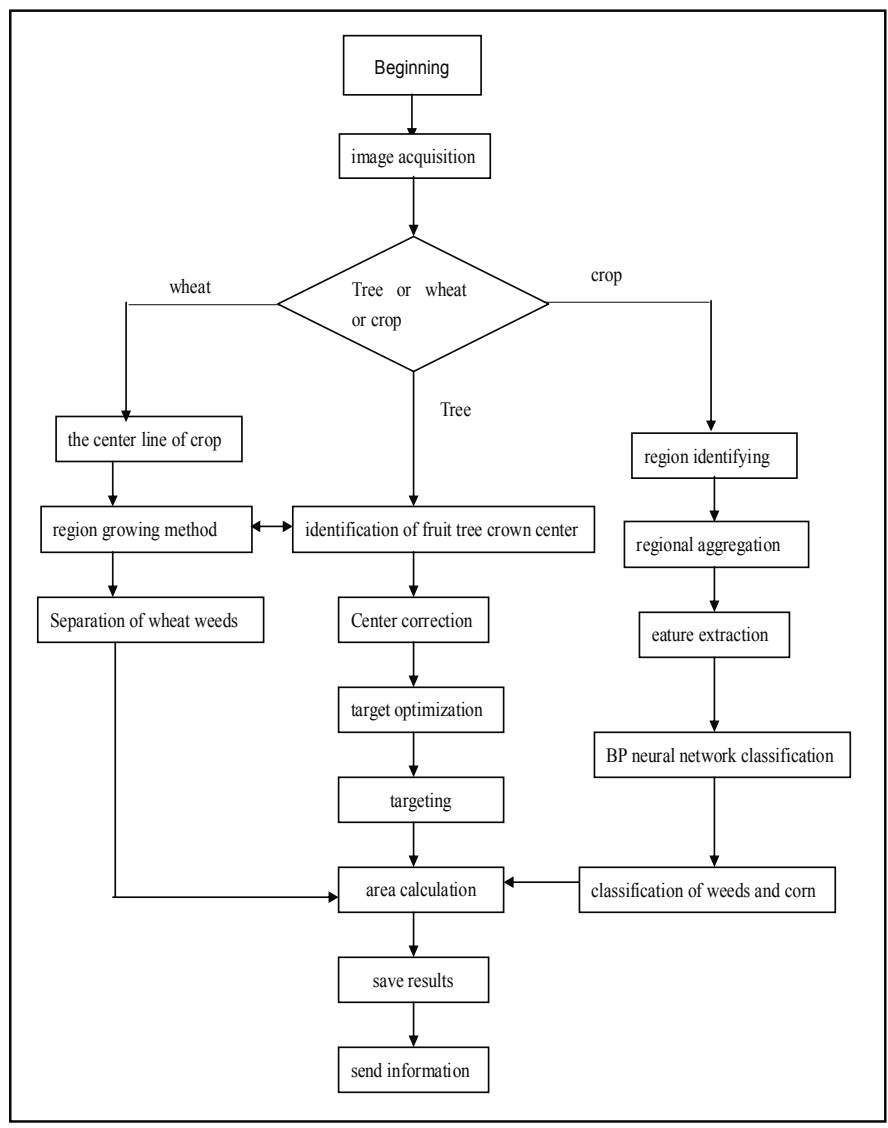

Fig. 1. Total system flow chart 
System software build platform as follow:

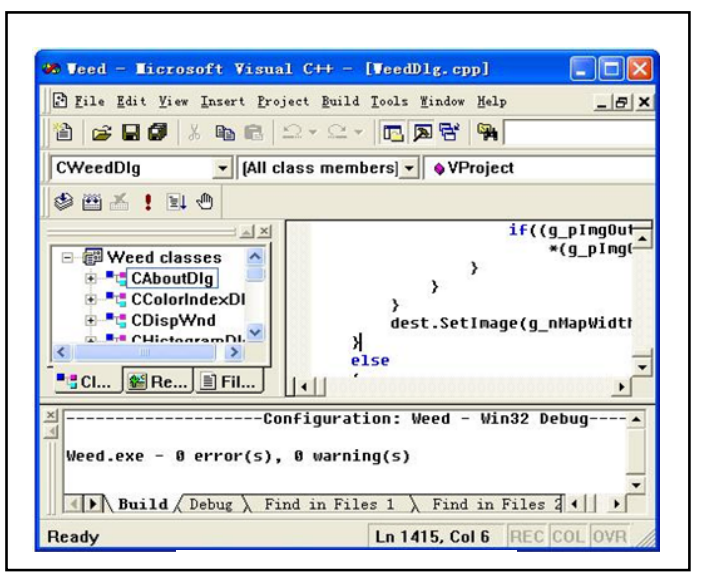

Fig. 2. System build platform

\section{Summary}

This article introduce the identification of software systems. The software system was designed with modular and class ideas. It consisted of five modules: module of image process, module of identifing the crop from the background of elaphic, module of target recognition and classification of treatment, module of intelligent decision-making and helpness. The system has fault-tolerant function and automatically judges the input of image or not, automatically detects input of camera and terminal setting, connection situation of singlechip, creating executable file, breaking away from $\mathrm{VC}++$ then direct running. Limiting surface of software system was friendly, simple clear and satisfy the real-time processing the request.

\section{References}

1. Won, S.L.: Robotic weed control system for tomatoes using machine vision system and precision chemical application. An ASAE Meeting Presentation 3093, 1-14 (1997)

2. Sheng, L.M.: Corn seedling weed identification in machine vision research Yang Ling: Northwest A\&F University, 3 (2002)

3. Paice, M.E., Miller, P.C.H., Bodle, J.D.: An experimental sprayer for the spatially selective application of herbicides. J. Agric-Eng. Res. 60(2), 107-116 (1995)

4. Wei, Z.: Research on the Technology of PWM Variable Sprayer, p. 4. Jiangsu University, Zhen Jiang (2006)

5. Kenzel, M.: United State Patent (December 1, 1998) 
6. Maocheng, Z., Jiaqiang, Z.: Tree crown recognition and precision toward-target pesticide application. Transactions of The Chinese Society of Agricultural Engineering (6), 150-153 (2003)

7. Qiu, B., Li, H., Wu, C.: On variable-rate spraying equipment and its key technology. Journal of Jiangsu University (National Science Edition) 98, 97-98 (2004)

8. Feng, G.Y.: Indoor Pesticide Smart-Targeting Application Simulation System Based on Machine Vision Master thesis]Nanjing Forestry University (2003) 